\title{
MICROSCALE SURFACE ENERGY PROPERTIES FOR ENHANCED SURFACE CONDENSATION AND SAMPLING OF EXHALED BREATH METABOLITES
}

\author{
Hamzeh K. Bardaweel ${ }^{l}$, Konstantin Zamuruyev ${ }^{l}$, Jean-Pierre Delplanque ${ }^{l}$, Nicholas J. Kenyon ${ }^{l}$, \\ Christopher Carron ${ }^{2}$, Oliver Brand ${ }^{2}$, Cristina E. Davis ${ }^{1 *}$ \\ ${ }^{1}$ University of California, Davis, CA, USA \\ ${ }^{2}$ Georgia Institute of Technology, Atlanta, GA, USA
}

\begin{abstract}
A MEMS-based exhaled breath sampler for the capture of both volatile and non-volatile biomarker metabolites is presented. The surface of the sampler is made superhydrophobic to enhance dropwise condensation and patterned with a radially-distributed wettability gradient that routes exhaled breath condensate droplets toward a central collection point. Results show that the surface of the sampler promotes dropwise condensation, and enables a freeenergy-driven mechanism to collect exhaled breath condensate from the surface. Contact angles, measured from the most hydrophobic to the least hydrophobic region, range from 157.0 to 126.8 degrees. Maximum contact angle hysteresis is approximately 1.8 degrees. Droplets deposited on the surface move at a rate of $6 \mathrm{~mm} / \mathrm{sec}$ towards the central collection point.
\end{abstract}

\section{INTRODUCTION}

Exhaled human breath analysis has become attractive as a diagnostic tool for various diseases including cancer, asthma, and respiratory infections [1-3]. One of the technological challenges for collecting exhaled breath samples from subjects is the design of an efficient and reliable breath sampler [4, 5]. Exhaled breath is usually collected with commercially available collection devices, such as the EcoScreen [6] and Rtube ${ }^{\mathrm{TM}}$ [7]. The available breath sampling technologies are power hungry, bulky, and show variability in performance [5]. There is a need for portable, efficient, and robust point-of-care breath samplers. Here we report our efforts toward fabricating a MEMS-based breath sampler for both volatile and non-volatile biomarker metabolite capture.

\section{DESIGN OF SAMPLER SURFACE}

Compared to currently used breath samplers, our MEMSbased sampler enhances the condensation process. It enables a free-energy-driven mechanism to collect exhaled breath condensate from a central point of the surface. The design of the MEMS-based sampler utilizes specifically-tailored superhydrophobic surfaces and a wettability gradient. The surface of the sampler is made superhydrophobic to promote dropwise condensation over filmwise condensation. The dropwise mode is preferable because it allows one order of magnitude larger coefficients of heat transfer between the surface and the exhaled breath gas [8]. The surface of the sampler is also patterned with a spatially-distributed wettability gradient that provides a freeenergy driven mechanism to route exhaled breath condensate droplets toward the collection point. In addition, the wettability gradient contributes to maintaining dropwise condensation on the sampler surface by continuously removing droplets from the surface, and thus freeing prior nucleation sites for new droplets to nucleate.

The wettability of a flat surface is described by Young's equation

$$
\cos \theta_{e}=\frac{\gamma_{S V}-\gamma_{S L}}{\gamma_{L V}}
$$

where $\theta_{e}$ is the equilibrium contact angle and $\gamma_{S V}, \gamma_{S L}$, and $\gamma_{L V}$ are the solid-vapor, solid-liquid, and the liquid-vapor interfacial free energies per unit area, respectively. Wenzel [9] proposed a mathematical model to predict the contact angle of a droplet on a rough surface, given by

$$
\cos \theta_{r}^{W}=r \cos \theta_{e}
$$

where $r$ is a roughness factor defined as the ratio of the actual area of the rough surface to the projected area. Wenzel's model assumes that the liquid completely fills the grooves of the rough surface. Cassie [10] proposed another mathematical model originally developed to describe the contact angle of a droplet on a chemically non-uniform surface, that can also be used to describe the contact angle of a droplet on a rough surface [11]

$$
\cos \theta_{r}^{C}=f_{s}\left(1+\cos \theta_{e}\right)-1
$$

where $f_{s}$ is the ratio of the area of the rough surface contacting the droplet to the projected area. The Cassie model assumes that the liquid is completely suspended by the micro-patterns.

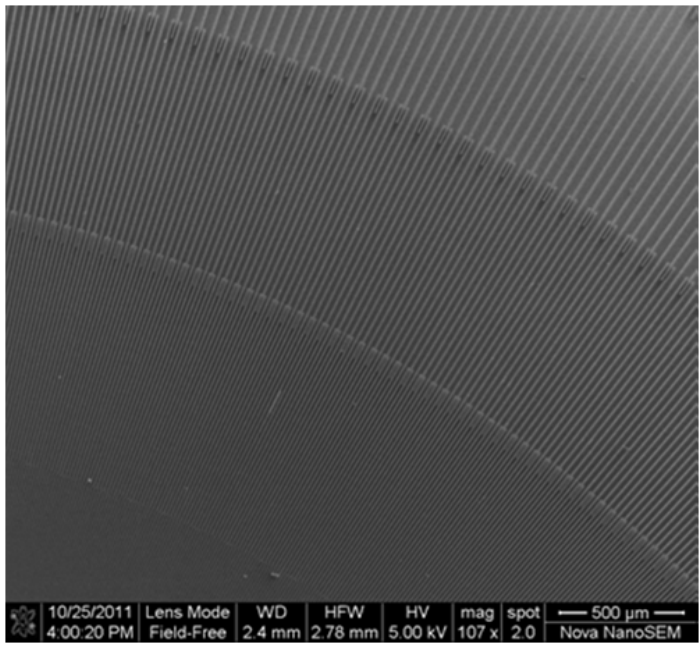

Figure 1. SEM image of the micropatterned sample surface.

Transport of droplets on graded surfaces has been investigated as a method of free-energy-driven droplet movement [12, 13]. Here, the presence of a surface wettability gradient induces the net mass transport of droplets. A droplet tends to move towards the more wettable side if it experiences an imbalance in surface tension forces across its edges, i.e. across the two opposite sides of the liquid-solid contact lines. Wettability gradients can be used to remove droplets from the surface by creating a spatial variation in the physical or chemical properties of the surface.

As the droplet moves along the surface, a resistance force is developed typically attributed to the presence of local defects [11]. The resistance force is measured by contact angle hysteresis 


$$
\left(\cos \theta_{R}-\cos \theta_{A}\right)=\frac{m g(\sin \alpha)}{\omega \gamma_{L V}}
$$

where contact angle hysteresis $\left(\theta_{R}-\theta_{A}\right)$ is defined as the difference between receding $\theta_{R}$ and advancing $\theta_{A}$ contact angles, $g$ is the acceleration of gravity, $\alpha$ is the minimum angle of tilt at which a droplet will spontaneously move, and $m$ and $\omega$ are the mass and width of the droplet base, respectively [14]. Thus a successful, free-energy driven droplet transport on roughened surface requires creating an imbalance in capillary forces across the edges of the droplet, and minimizing contact angle hysteresis.

Dropwise condensation on superhydrophobic surfaces has been investigated thoroughly by many research groups [15-18]. Dropwise condensation is the preferred regime of condensation due to its higher rate of heat transfer, compared to the filmwise condensation regime [8]. However, initiating and maintaining dropwise condensation has proven challenging $[15,18,19]$. While initiating dropwise condensation requires non-wettable surfaces, maintaining dropwise condensation requires continuous removal of small droplets from the surface. During filmwise condensation the condensed liquid tends to wet the surface, and a film of liquid is formed on the surface by droplets coalescence. The presence of the liquid film significantly reduces heat transfer across the surface and, consequently, the rate of condensation decreases as well. In configurations where wettability is poor, the formation of a liquid film is impeded, and the surface is covered with a distribution of droplets with various sizes; this is dropwise condensation. In this regime, heat transfer between the surface and the humid air is only affected where droplets are present. The larger droplets with sizes greater than $10 \mu \mathrm{m}$ [20] have a large thermal resistance and thus behave locally like a liquid film, significantly hindering heat transfer. Very often dropwise condensation turns quickly into filmwise condensation, reducing the effectiveness of heat transfer and thus the condensation process.

\section{SAMPLER SURFACE FABRICATION}

Both chemical composition and physical roughness of the surface contribute to its wettability. In this work, the surface is patterned with micro-fabricated features, namely etched grooves, to increase its hydrophobicity. The wettability gradient is obtained by gradually varying the roughness of the micro-patterned surface. The surface is subsequently coated with a low energy interface material to make it superhydrophobic. Silicon is chosen as the material of the sampler surface because of its high thermal conductivity and its adaptability with microfabrication techniques. The surface is patterned using contact photolithography and deep reactive ion etching is used to etch the characteristic groove/ridge structure. The widths of the ridges and grooves modulate the surface wettability and, thus, are changed radially to establish a wettability gradient in the radial direction of the circular sampler geometry. Polybutadiene (Aldrich, MW= $420000,36 \%$ cis 1,4 addition, 55\% trans 1,4 addition, 9\% 1,2 addition) is then dissolved in toluene $(99.5 \%$ purity) at a concentration of $5 \%(\mathrm{w} / \mathrm{w})$ [21]. The surface of the sampler is spin-coated with the prepared solution and annealed in a vacuum oven to remove entrapped solvent. The coated surface is plasma treated in a vacuum chamber. Lastly the plasma fluorinated polybutadiene film is treated in a vacuum chamber. Further details on plasma fluorination are provided by Woodward et al. [21, 22]. Figure 1 shows a SEM picture of the micro-patterned sampler surface. The surface consists of micropatterned concentric regions. Each region consists of a periodic arrangement of radially patterned ridges and grooves with the and grooves decreasing) toward the center of the surface.

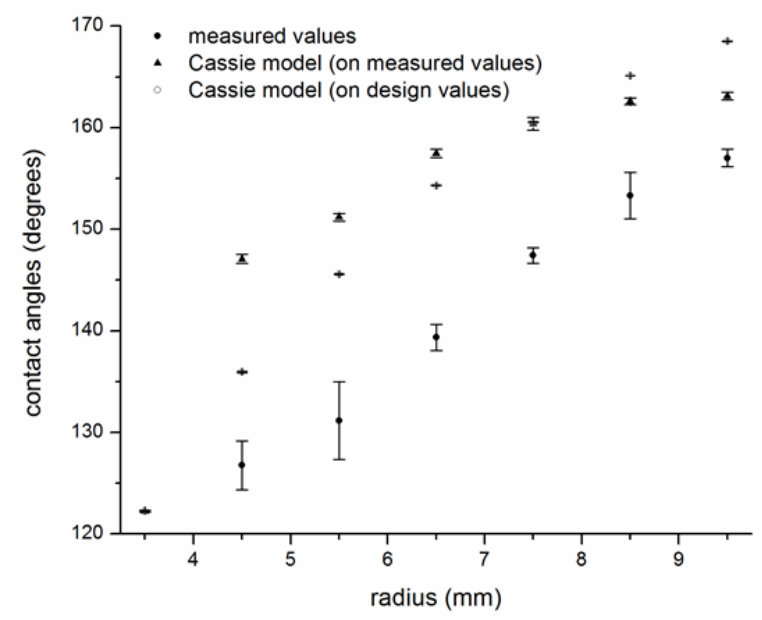

Figure 2. Contact angle distribution on the surface of the sampler.

Thus, the surface roughness is tuned by increasing the number of walls and grooves toward the center. The collection point is an un-patterned circular area at the center, $8 \mathrm{~mm}$ in diameter. The grooves are approximately $60 \mu \mathrm{m}$ deep. The sampler is $20 \mathrm{~mm}$ in diameter and fabricated on $500 \mu \mathrm{m}$ thick silicon wafers.

\section{RESULTS AND DISCUSSION}

In characterizing the surface of the sampler we designed two sets of experiments. The first set of experiments investigated the movement of droplets on the surface of the sampler. In Figure 2, the contact angles of water droplets deposited on the surface of the sampler were measured with a goniometer (Ramé-hart Model 250). Both measured and Cassie-modeled contact angles are shown. Here, contact angles predicted by the Wenzel model (not shown) deviate sharply from measured data. This can be explained if the droplets deposited on the surface of the sampler are lifted by the micro-patterns (Cassie model). The plot shows that contact angles predicted by the Cassie model show similar trends to measured contact angles. In calculating the contact angles predicted by the Cassie model, the actual dimensions of the fabricated grooves/ridges were measured (with an SEM Nova ${ }^{\mathrm{TM}}$ NanoSEM 230) and substituted in the model. The wettability gradient is distributed between the outer edge of the sampler surface, which is the most hydrophobic region, and the center collection point of the sampler, which is the least hydrophobic region. The measured contact angles decreased gradually from 157.0 to 126.7 degrees toward the center of the sampler surface. The maximum measured contact angle hysteresis $\left(\theta_{R}-\theta_{A}\right)$ is approximately 1.8 degrees. Thus, resistance for movement of droplets deposited on the surface is minimized.

Figure 3 shows an example of a video capture sequence of a series of purified water droplets deposited with a syringe on the surface of the sampler. The droplets size ranged between 2-10 $\mu \mathrm{L}$. As the droplet was deposited on the surface it moved along the wettability gradient toward the center of the surface driven by capillarity. To successfully maintain the mobility of the droplet on the surface, the difference in capillary forces must overcome the induced resistance force, described by equation 4 .

In the second set of experiments the behavior of the sampler surface under condensation conditions was investigated. In condensation tests, the surface of the sampler was actively cooled with a thermoelectric element (Custom Thermoelectric, model number: 19811-9L31-02CN1). The average surface temperature was $16{ }^{\circ} \mathrm{C}$. 

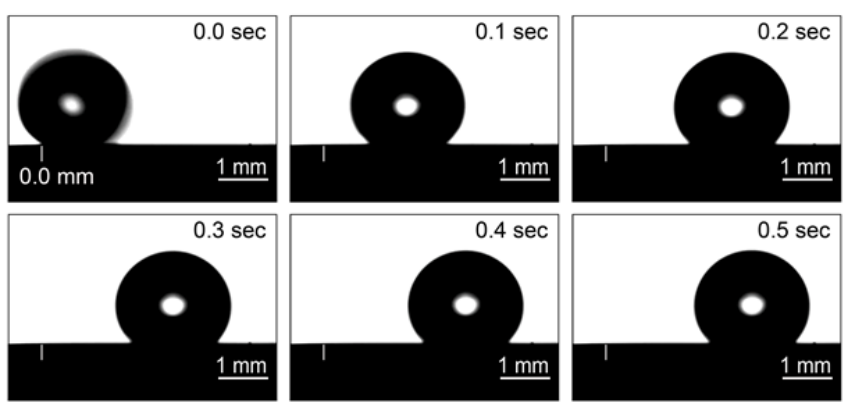

Figure 3. Radial movement of the droplet.

The test was done at atmospheric pressure and ambient temperature $\left(22.2{ }^{\circ} \mathrm{C}\right)$. The relative humidity of the room air was measured at $\phi=48 \%$. Video imaging of the condensation process was captured with a camera (SENTECH) attached to an optical microscope (CARL ZEISS, model number: 452825). The observed condensation process is shown Figure 5. Initially, droplets nucleate on the surface without significant interactions between them. As droplets grow, some of them coalesce and continue to grow in size to form larger droplets. After nucleation and initial growth, droplets merge at their contact lines with other droplets. As droplets coalesce, new nucleation sites are available - allowing for new nuclei to grow. As condensation progresses, droplets continue growing in size. Once they reach a critical size (i.e. the droplets "feel" the wettability gradient across their edges), they move in a directional manner along the wettability gradient.

The ability of the super-hydrophobic micro-patterned surface to promote dropwise condensation is demonstrated in Figure 5. This shows the sampler surface at the end of a condensation test. Two forms of sweeping actions took place during the condensation process. First, we observe "local sweeping" when nucleated droplets sweep the surface as they grow in size and coalesce. Secondly, "radial sweeping" is observed as larger droplets sweep the surface and swallow smaller droplets as they move along the wettability gradient toward the collection point. Radial sweeping clears the smaller droplets from the surface of the sampler, and therefore creates new sites for droplet nucleation and growth.

\section{CONCLUSIONS}

In this article, we have performed initial characterization of a MEMS-based exhaled breath sampler. The surface of the sampler is made superhydrophobic and promotes dropwise condensation over filmwise condensation. The surface of the sampler is also patterned with a spatially-distributed wettability gradient that provides a free-energy driven mechanism to route exhaled breath condensate droplets toward the collection point at the center of the condenser. Maximum measured contact angle hysteresis is approximately 1.8 degrees. Droplets deposited on the surface move in directional manner, toward the center of the sampler. Upon condensation, droplets nucleate and coalesce. As they grow, they move and sweep the surface, swallowing smaller droplets. Large droplets move along the pre-designed wettability gradient, toward the center of the sampler surface. New nucleation sites become continuously available as a result of droplets removal, allowing for new nuclei to grow. Since heat transfer is mostly suppressed at locations where droplets have diameters larger than $10 \mu \mathrm{m}$, this method of removing droplets from the surface during dropwise condensation is effective.
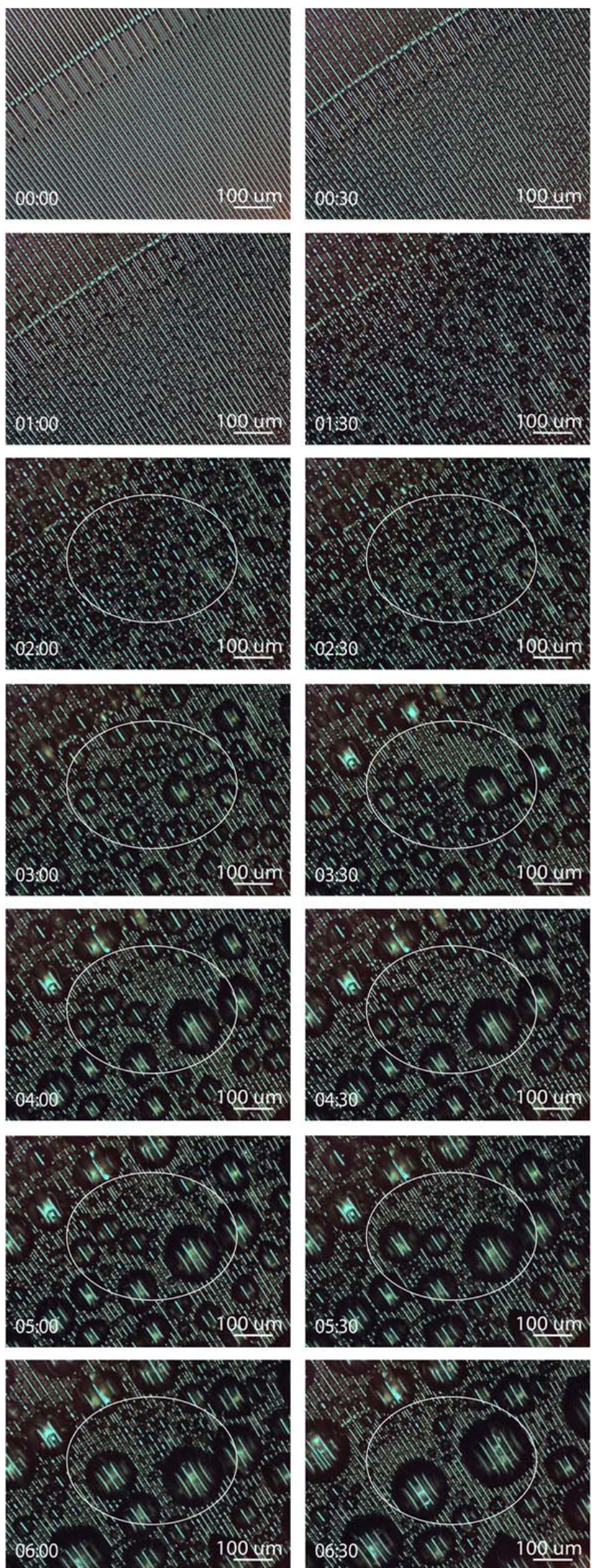

Figure 4. Condensation cycle: droplet nucleation, growth, local sweeping, radial sweeping, new sites are available for nucleation, and a new condensation cycle starts. 


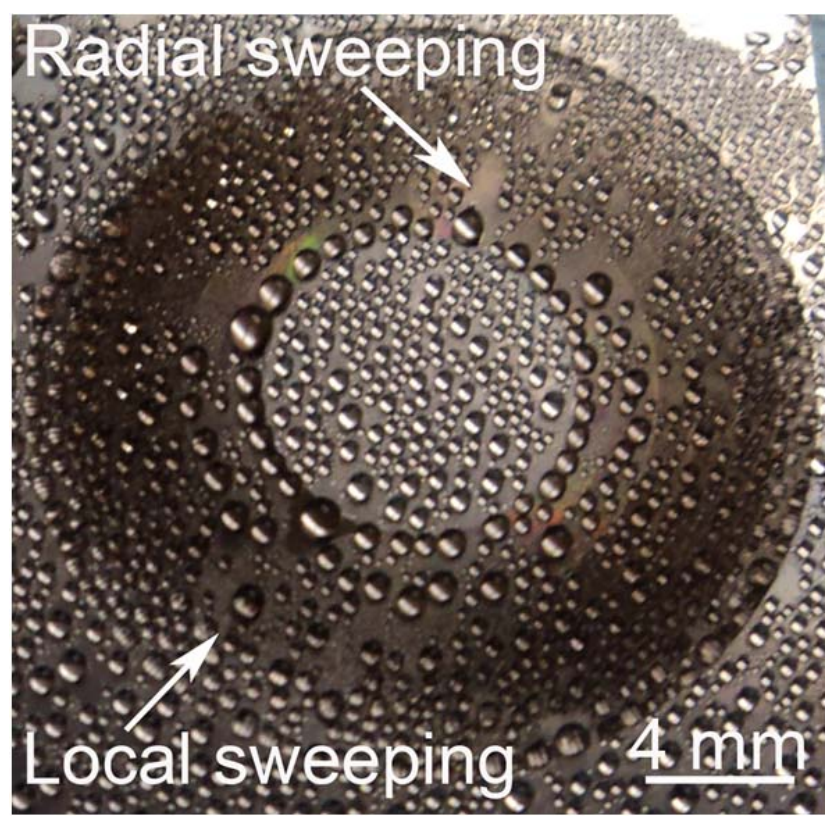

Figure 5. Sampler surface at the end of the condensation test.

\section{REFERENCES:}

[1] Manolis, A., "The diagnostic potential of breath analysis." Clinical Chemistry, 1983. 29(1): p. 5-15.

[2] Kharitonov, S.A. and P.J. Barnes, "Biomarkers of some pulmonary diseases in exhaled breath." Biomarkers, 2002. 7(1): p. 1-32.

[3] Horvath, I., Hunt, J., Barnes, P. J., "Exhaled breath condensate: methodological recommendations and unresolved questions." European Respiratory Journal, 2005. 26(3): p. 523-548.

[4] Liu, J., Conrad, D. H., Chow, S., Tran, V. H., Yates, D. H., Thomas, P. S., "Collection devices influence the constituents of exhaled breath condensate." European Respiratory Journal, 2007. 30(4): p. 807-808.

[5] Rosias, P. P., Robroeks, C. M., Kester, A., den Hartog, G. J., Wodzig, W. K., Rilkers, G. T., Zimmermann, L. J., van Schayck, C. P., Joebsis, Q., Dompeling, E., "Biomarker reproducibility in exhaled breath condensate collected with different condensers." European Respiratory Journal, 2008. 31(5): p. 934-942.

[6] Carpagnano, G. E., Kharitonov, S. A., Foschino-Barbaro, M. P.Resta, O., Gramiccioni, E., Barnes, P. J., "Increased inflammatory markers in the exhaled breath condensate of cigarette smokers." European Respiratory Journal, 2003. 21(4): p. 589-593.

[7] Esther, C. R., Jasin, H. M., Collins, L. B., Swenberg, J. A., Boysen, G., "A mass spectrometric method to simultaneously measure a biomarker and dilution marker in exhaled breath condensate." Rapid Communications in Mass Spectrometry, 2008. 22(5): p. 701-705.

[8] Rose, J.W., "Dropwise condensation theory and experiment: a review." Proceedings of the Institution of Mechanical Engineers Part A-Journal of Power and Energy, 2002. 216(A2): p. 115-128.

[9] Wenzel, R.N., "Surface Roughness and Contact Angle." Journal of Physical and Colloid Chemistry, 1949. 53(9): p. 1466-1467.

[10] Cassie, A.B.D., "Contact Angles." Discussions of the Faraday Society, 1948. 3: p. 11-16.
[11] Berthier, J., "Microdrops and digital microfluidics." Micro \& nano technologies 2008, Norwich, NY: William Andrew Pub. xviii, $441 \mathrm{p}$.

[12] Chaudhury, M.K. and G.M. Whitesides, "How to Make Water Run Uphill." Science, 1992. 256(5063): p. 1539-1541.

[13] Callies, M. and D. Quere, "On water repellency." Soft Matter, 2005. 1(1): p. 55-61.

[14] Furmidge, C.G., "Studies at Phase Interfaces .1. Sliding of Liquid Drops on Solid Surfaces and a Theory for Spray Retention." Journal of Colloid Science, 1962. 17(4): p. 309-\&.

[15] Wier, K.A. and T.J. McCarthy, "Condensation on ultrahydrophobic surfaces and its effect on droplet mobility: Ultrahydrophobic surfaces are not always water repellant." Langmuir, 2006. 22(6): p. 2433-2436.

[16] Dorrer, C. and J. Ruhe, "Condensation and wetting transitions on microstructured ultrahydrophobic surfaces." Langmuir, 2007. 23(7): p. 3820-3824.

[17] Narhe, R.D. and D.A. Beysens, "Nucleation and growth on a superhydrophobic grooved surface." Physical Review Letters, 2004. 93(7).

[18] Boreyko, J.B. and C.-H. Chen, "Self-Propelled Dropwise Condensate on Superhydrophobic Surfaces." Physical Review Letters, 2009. 103(18): p. 184501.

[19] Varanasi, K. K., Hsu, M., Bhate, N., Yang, W. S., Deng, T., "Spatial control in the heterogeneous nucleation of water." Applied Physics Letters, 2009. 95(9).

[20] Graham, C. and P. Griffith, "Drop Size Distributions and Heat-Transfer in Dropwise Condensation." International Journal of Heat and Mass Transfer, 1973. 16(2): p. 337-346.

[21] Woodward, I. S., Schofield, W. C. E., Roucoules, V., Bradley, T. J., Badyal, J. P. S., "Micropatterning of plasma fluorinated super-hydrophobic surfaces." Plasma Chemistry and Plasma Processing, 2006. 26(5): p. 507-516.

[22] Woodward, I., Schofield, W. C. E., Roucoules, V., Badyal, J. P. S., "Super-hydrophobic surfaces produced by plasma fluorination of polybutadiene films." Langmuir, 2003. 19(8): p. $3432-3438$.

\section{CONTACT}

* C.E. Davis, tel: +1 530754 9004; cedavis@ucdavis.edu

\section{ACKNOLWLEDGEMENTS}

This work was generously supported by The Hartwell Foundation [CD, JPD]. Partial support was also provided by the United States Air Force Research Laboratory through contract FA8650-08-C-6832 and UES, Inc. [CD, JPD, OB]. This work was also supported in part by the National Center for Research Resources (NCRR) under Grant UL1RR024146-06 [CD], a component of the National Institutes of Health (NIH). Graduate fellowship support [KZ] was provided by award number P42ES004699 from the National Institute of Environmental Health Sciences (NIEHS).

The contents of this manuscript are solely the responsibility of the authors and do not necessarily represent the official view of the funding agencies. 